J-J. Sinou and E. Jacquelin, Influence of polynomial chaos expansion order on an uncertain asymmetric rotor system response, Mechanical Systems and Signal Processing, 50,718-731, 2015.

doi:10.1016/j.ymssp.2014.05.046

\title{
Influence of polynomial chaos expansion order on an uncertain asymmetric rotor system response
}

\author{
J.-J. Sinou ${ }^{\mathrm{a}, *}$, E. Jacquelin ${ }^{\mathrm{b}, \mathrm{c}, \mathrm{d}}$ \\ ${ }^{a}$ École Centrale de Lyon, LTDS, UMR CNRS 5513, F-69134, Écully - France \\ ${ }^{b}$ Université de Lyon, F-69622, Lyon, France \\ ${ }^{c}$ Université Claude Bernard Lyon 1, Villeurbanne \\ ${ }^{d}$ IFSTTAR, UMR-T9406, LBMC Laboratoire de Biomécanique et Mécanique des chocs, F69675, Bron
}

\begin{abstract}
A stochastic harmonic balance method with a recursive procedure is developed to evaluate the steadystate response of a rotor system with uncertain stiffness and asymmetric coupling that involves timedependent terms. The Polynomial Chaos (PC) expansion is proposed to evaluate the mean and the standard deviation of the responses and the harmonic amplitudes of orders 1 to 4 involving 288 degrees-of-freedom. To determine the coefficients of the expansion requires to solve a large system of equations. To achieve this and to avoid numerical problems related to the size of the system, the first contribution of the study consists of a recursive evaluation of the polynomial chaos coefficients to be able to estimate the stochastic response for a high order of the Polynomial Chaos expansion.

From the methodology implemented, the steady-state responses and $n \times$ harmonic components (for $n=1,2,3$ and 4 in the present study) of the asymmetric rotor with uncertainty are evaluated for several PC orders. Then, the second main contribution focuses on a clarification and analysis of the use of Polynomial Chaos expansion around the critical speeds. First, it is observed that the convergence is slow: the response obtained with 30 PCs is twice the one obtained with 200 PCs. Second, it is noted that the parity of the PC order has a strong influence on the response level: whereas two responses obtained with two consecutive even PC orders (or two consecutive odd PC orders) are almost the same for a given rotation speed, the ratio of the responses evaluated with two consecutive PC orders (one even order and one odd order) may be large (e.g. oscillations between two consecutive PC orders greater than 10 are noticeable if the $\mathrm{PC}$ order is about 30).
\end{abstract}

\section{Introduction}

In recent decades, there is a strong growth in the analysis of the dynamic response of large mechanical systems with uncertainties. One of the most commonly used statistical approach is the Monte Carlo (MC) method, which includes uncertainties in the deterministic problem. The MC method

\footnotetext{
*Corresponding author: jean-jacques.sinou@ec-lyon.fr, Tel: +33472186466

Email addresses: jean-jacques.sinou@ec-lyon.fr (J.-J. Sinou), eric.jacquelin@univ-lyon1.fr (E. Jacquelin)
} 
generates random realizations for independent samples of the random parameters and utilizes repetitive deterministic solvers for each realization. One of the major drawbacks of this approach lies in the computational cost and therefore the need to consider a large number of samples to obtain the convergence of the statistical response. Furthermore, other numerous methods have been developed to quantify physical uncertainties in a variety of computational problems such as the perturbation methods [1] that are based on the development of random quantities in Taylor or Neumann series $[2,3]$. These approaches are efficient only for small random fluctuations and seem not suitable to solve a problem of dynamics for frequencies near resonance. Then the Polynomial Chaos (PC) expansions $[4,5]$ that can be used to characterize the solution of equations with random parameters has received increasing attention over the past few years. Some recent works proposed to extend the Polynomial Chaos expansion by introducing the generalized Polynomial Chaos $[6,7,8]$ or adaptive strategies based on the Multi-Element generalized Polynomial Chaos (MEgPC) [9]. The main idea of this last strategy is based on the fact that it can be more efficient to use a piecewise expansion with low degree of PC expansion rather than a one element expansion with high polynomial degree. The efficiency of the proposed methodology was demonstrated through several example for practical mechanical systems $[10,11]$.

However, the effects of PC expansion on the response level and the convergence of the stochastic response remain misunderstood and most of the numerical studies using classical PC expansion fail to give an accurate response over all the frequency range of interest. For example, the PC expansion has been previously proposed in $[12,13,14,15]$ in order to determine the dynamic response of rotating systems subjected to uncertainties and single or multiple excitations. Even if the results of the Frequency Response Function for both the mean and standard deviation were in agreement with the classical Monte Carlo simulations, the results were not convincing at critical speeds: effectively it can be noted that the mean and variance were not properly estimated around the critical speeds where an important overestimation of levels was observed.

So the major contribution and the main objective of the present study propose a new methodology based on the PC expansion to calculate an accurate stochastic response of large mechanical systems and to discuss the need to consider the high PC orders to obtain an accurate solution. The novelty in the attempted work appears in the formulation of a recursive procedure to expand the stochastic response on very high PC orders. Thus the influence of the PC expansion on the response level, the convergence of the stochastic response and the role of the parity in the PC order will be discussed. To illustrate this, an example, which corresponds to an asymmetric rotor system with uncertainty and parametric terms, will be addressed.

To achieve the previous objectives, the paper is organized as follows: firstly, the general equations of the asymmetric rotor system with uncertainty are presented. Then, the expansion of the stochastic 
response as a stochastic truncated Fourier series is detailed: the mean and the standard deviation of the responses are evaluated by using a PC expansion with the combination of the Harmonic Balance Method. Moreover, a recursive procedure is developed to estimate the stochastic steady-state response of large system of equations with high PC orders. Finally, the influence of the PC expansion and the convergence of the mean and the standard deviation of the $n \times$ harmonic components (for $n=1,2,3$ and 4 in the present study) and the global steady-state response of asymmetric rotor are investigated.

\section{Asymmetric rotor with uncertain parameters}

\subsection{General equation of the asymmetric rotor system}

The layout of the rotor system under consideration is shown in Figure 1. This rotor that consists of a steel shaft of $0.7 \mathrm{~m}$ long and $0.04 \mathrm{~m}$ diameter with one disc is simply supported by two bush bearings. Moreover, an asymmetric coupling is added on the right end of the shaft. All the values of the material properties and dimensions of the rotor are given in Table 1.

The rotor is discretized into 7 Timoshenko beam finite elements (length of $0.1 \mathrm{~m}$ ), with four degrees of freedoms at each node (two lateral displacements and two rotations), the axial and torsional degrees of freedom being not considered. After assembling the various shaft elements, the rigid disc, the bearing supports and the asymmetric coupling, the equations of the rotor system can be written as [16]

$$
\mathbf{M} \ddot{\mathbf{x}}+(\mathbf{C}+\omega \mathbf{G}) \dot{\mathbf{x}}+\left(\mathbf{K}_{\mathrm{sh}}+\mathbf{K}_{\mathrm{sup}}+\mathbf{K}_{\mathbf{f}}^{\mathbf{c}}+\mathbf{K}_{\mathbf{c}}^{\mathbf{c}} \cos (2 \omega t)+\mathbf{K}_{\mathbf{s}}^{\mathbf{c}} \sin (2 \omega t)\right) \mathbf{x}=\mathbf{f}+\mathbf{q}
$$

where $\mathbf{x}$ defines the steady-state response of the rotor system. The size of the vector $\mathbf{x}$ equals 32 . $\mathbf{f}$ and $\mathbf{q}$ contain the gravitational and unbalance forces, respectively. The mass matrix $\mathbf{M}$ includes the mass matrices of the shaft, the rigid disc and the coupling. The matrix $\mathbf{K}_{\text {sh }}$ (resp. $\mathbf{K}_{\mathrm{sup}}$ ) is the stiffness matrix of the shaft (resp. the supports) and contains the circulatory matrix, which accounts for the shafts internal damping.

The matrix $\mathbf{C}$ includes the effects of the shafts internal damping and damping of the supports. $\mathbf{G}$ is the gyroscopic matrix. Finally, the matrices $\mathbf{K}_{\mathbf{f}}^{\mathbf{c}}, \mathbf{K}_{\mathbf{c}}^{\mathbf{c}}$ and $\mathbf{K}_{\mathbf{s}}^{\mathbf{c}}$ correspond to the contributions of the asymmetric coupling. These global stiffness matrices can be written as

$$
\operatorname{diag}\left(\mathbf{K}_{\mathbf{f}}^{\mathbf{c}}\right)=\left(\begin{array}{lll}
\mathbf{0} \cdots \mathbf{0} & \mathbf{k}_{\mathbf{f}}^{\mathbf{c}}
\end{array}\right) \quad ; \quad \operatorname{diag}\left(\mathbf{K}_{\mathbf{c}}^{\mathbf{c}}\right)=\left(\begin{array}{lll}
\mathbf{0} \cdots \mathbf{0} & \mathbf{k}_{\mathbf{c}}^{\mathbf{c}}
\end{array}\right) \quad ; \quad \operatorname{diag}\left(\mathbf{K}_{\mathbf{s}}^{\mathbf{c}}\right)=\left(\begin{array}{lll}
\mathbf{0} \cdots \mathbf{0} & \mathbf{k}_{\mathbf{s}}^{\mathbf{c}}
\end{array}\right)
$$

where $\mathbf{0}$ defines the $4 \times 4$ null matrix (for the corresponding four degrees of freedoms at each node). Expressions of $\mathbf{k}_{\mathbf{f}}^{\mathbf{c}}, \mathbf{k}_{\mathbf{c}}^{\mathbf{c}}$ and $\mathbf{k}_{\mathbf{s}}^{\mathbf{c}}$ are given by [17]

$$
\mathbf{k}_{\mathbf{f}}^{\mathbf{c}}=\left[\begin{array}{cccc}
\frac{1}{2}\left(k_{x x}+k_{y y}\right) & \frac{1}{2}\left(k_{x y}-k_{y x}\right) & 0 & 0 \\
\frac{1}{2}\left(k_{y x}-k_{x y}\right) & \frac{1}{2}\left(k_{x x}+k_{y y}\right) & 0 & 0 \\
0 & 0 & k_{\theta \theta} & 0 \\
0 & 0 & 0 & k_{\phi \phi}
\end{array}\right]
$$




$$
\begin{gathered}
\mathbf{k}_{\mathbf{s}}^{\mathbf{c}}=\left[\begin{array}{cccc}
\frac{1}{2}\left(k_{x y}+k_{x y}\right) & \frac{1}{2}\left(k_{y y}-k_{x x}\right) & 0 & 0 \\
\frac{1}{2}\left(k_{y y}-k_{x x}\right) & -\frac{1}{2}\left(k_{x y}+k_{y x}\right) & 0 & 0 \\
0 & 0 & 0 & 0 \\
0 & 0 & 0 & 0
\end{array}\right] \\
\mathbf{k}_{\mathbf{s}}^{\mathbf{c}}=\left[\begin{array}{cccc}
\frac{1}{2}\left(k_{x x}-k_{y y}\right) & \frac{1}{2}\left(k_{x y}+k_{y x}\right) & 0 & 0 \\
\frac{1}{2}\left(k_{x y}+k_{y x}\right) & \frac{1}{2}\left(k_{y y}-k_{x x}\right) & 0 & 0 \\
0 & 0 & 0 & 0 \\
0 & 0 & 0 & 0
\end{array}\right]
\end{gathered}
$$

where the physical values of the asymmetric coupling are given in Table 2. Moreover, the mass of the asymmetric coupling is $2.1 \mathrm{~kg}$. The above equations of the rotor (1) have time-dependent coefficients (i.e. parametric terms) due to the variation of the contributions of the asymmetric coupling when the system rotates (i.e. $\mathbf{K}_{\mathbf{c}}^{\mathbf{c}} \cos (2 \omega t)$ and $\left.\mathbf{K}_{\mathbf{s}}^{\mathbf{c}} \sin (2 \omega t)\right)$.

[Figure 1 about here.]

[Table 1 about here.]

[Table 2 about here.]

\subsection{Uncertainties}

Let us consider the probability space that presents a model for the particular mechanical system under study. It consists of the triplet $(\Omega, \mathfrak{F}, P)$ where $\Omega$ is the event space, $\mathcal{F}$ is a $\sigma$-algebra on $\Omega$ and $P$ a probability measure. Consider a random variable $\xi$ defined on the probability space $(\Omega, \mathfrak{F}, P)$ and having a standard centered Gaussian distribution.

In this study, the Young modulus of the shaft is taken as random and defined by following

$$
E=\bar{E}\left(1+\delta_{E} \xi(\tau)\right)
$$

where $\bar{E}$ is the mean of Young modulus of the shaft and $\delta_{E}$ is the variation coefficient of the random Young modulus.

Consequently, the shaft stiffness matrix, $\mathbf{K}_{\text {sh }}$, is supposed to be random:

$$
\mathbf{K}_{\mathrm{sh}}(\tau)=\overline{\mathbf{K}}_{\mathrm{sh}}\left(1+\delta_{E} \xi(\tau)\right)
$$

where $\overline{\mathbf{K}}_{\text {sh }}$ is the mean shaft stiffness matrix. The deterministic case corresponds to $\delta_{E}=0$. 


\section{Stochastic response of the system}

Equation (1) has parametric terms due to the contribution of the coupling element. As previously explained in $[13,15]$, for rotating machines excited by unbalance and in which the stiffness coefficient varies sinusoidally, the steady-state response is periodic. So the stochastic response can be approximated by a stochastic truncated Fourier series of order $m$

$$
\mathbf{x}(\omega, t, \tau)=\mathbf{A}_{0}(\tau)+\sum_{h=1}^{m}\left(\mathbf{A}_{\mathbf{h}}(\tau) \cos (h \omega t)+\mathbf{B}_{\mathbf{h}}(\tau) \sin (h \omega t)\right)=\mathbf{x}_{\mathbf{0}}(\omega, t, \tau)+\sum_{h=1}^{m} \mathbf{x}_{\mathbf{h}}(\omega, t, \tau)
$$

with argument $\tau$ denoting the random character. $\omega$ defines the deterministic fixed rotational speed of the rotor system. $\mathbf{A}_{\mathbf{0}}(\tau), \mathbf{A}_{\mathbf{h}}(\tau)$ and $\mathbf{B}_{\mathbf{h}}(\tau)$ (with $h=1, \cdots, m$ ) define the unknown stochastic coefficients of the finite Fourier series. The number of harmonic coefficients, $m$, is selected on the basis of the number of significant harmonics expected in the stochastic response; in the present study, $m$ is equal to 4 due to previous results obtained for similar rotor system [13]. $\mathbf{x}_{\mathbf{h}}$ is the response of order $h($ for $h=1,2,3$ or 4$)$.

Equation (1) can then be rewritten on the stochastic Fourier basis such that

$$
\boldsymbol{\Lambda}(\tau) \Theta(\tau)=\boldsymbol{\Gamma}
$$

where $\boldsymbol{\Lambda}(\tau)$ is a random matrix and $\boldsymbol{\Gamma}$ is a deterministic vector: they will be defined in the next two subsections. $\boldsymbol{\Theta}(\tau)$ defines the stochastic truncated Fourier series such that

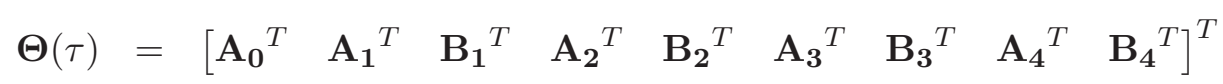

It can be noted that the size of vector $\Theta$ is equal to 288 (i.e. 32 degrees of freedom for the original system defined in equation (1) and a decomposition with 9 harmonic components for each degree of freedom of equation (1).

\subsection{Expression of $\boldsymbol{\Gamma}$}

It can be noted that the gravitational force $\mathbf{f}$ and the unbalance force $\mathbf{q}$ can be exactly defined by deterministic finite Fourier series (i.e. $\mathbf{f}=\mathbf{C}_{\mathbf{0}}^{\mathbf{f}}$ and $\mathbf{q}=\mathbf{C}_{\mathbf{1}}^{\mathbf{q}} \cos (\omega t)+\mathbf{S}_{\mathbf{1}}^{\mathbf{q}} \sin (\omega t)$ ). So the deterministic expression $\boldsymbol{\Gamma}$ can be defined by

$$
\boldsymbol{\Gamma}=\left[\begin{array}{llllll}
\mathbf{C}_{\mathbf{0}}^{\mathbf{f}^{T}} & \mathbf{C}_{\mathbf{1}}^{\mathbf{q}^{T}} & \mathbf{S}_{\mathbf{1}}^{\mathbf{q}^{T}} & \mathbf{0}^{T} & \cdots & \mathbf{0}^{T}
\end{array}\right]^{T}
$$

where $\mathbf{C}_{\mathbf{0}}^{\mathbf{f}}, \mathbf{C}_{\mathbf{1}}^{\mathbf{q}}$ and $\mathbf{S}_{\mathbf{1}}^{\mathbf{q}}$ are the vectors of the constant Fourier components of the gravitational force, the first-order periodic components in the frequency domain of the unbalance force, respectively. 


\subsection{Expression of $\boldsymbol{\Lambda}(\tau)$}

Expression $\boldsymbol{\Lambda}(\tau)$ is established by combining the stochastic truncated Fourier response $\mathbf{x}(\omega, \tau)$ and the equation of the rotor system and $\boldsymbol{\Lambda}(\tau)$ is written

$$
\boldsymbol{\Lambda}(\tau)=\boldsymbol{\Lambda}_{\mathbf{a}}(\tau)+\boldsymbol{\Lambda}_{\mathbf{b}}
$$

where $\boldsymbol{\Lambda}_{\mathbf{a}}(\tau)$ and $\boldsymbol{\Lambda}_{\mathbf{b}}$ are defined as:

$$
\begin{aligned}
& \boldsymbol{\Lambda}_{\mathbf{a}}(\tau)=\left[\begin{array}{ccccc}
\mathbf{K}_{\mathrm{sh}}(\tau)+\mathbf{K}_{\mathrm{sup}}+\mathbf{K}_{\mathbf{f}}^{\mathrm{c}} & \mathbf{0} & \mathbf{0} & \mathbf{0} & \mathbf{0} \\
0 & \lambda_{1} & 0 & 0 & 0 \\
0 & 0 & \lambda_{2} & 0 & 0 \\
0 & 0 & 0 & \lambda_{3} & 0 \\
0 & 0 & 0 & 0 & \lambda_{4}
\end{array}\right] \\
& \boldsymbol{\Lambda}_{\mathrm{b}}=\frac{1}{2}\left[\begin{array}{ccccccccc}
0 & \mathbf{0} & \mathbf{0} & \mathbf{K}_{\mathrm{c}} & \mathbf{K}_{\mathrm{s}} & \mathbf{0} & \mathbf{0} & \mathbf{0} & \mathbf{0} \\
\mathbf{0} & \mathbf{K}_{\mathrm{c}} & \mathbf{K}_{\mathrm{s}} & \mathbf{0} & \mathbf{0} & \mathbf{K}_{\mathrm{c}} & \mathbf{K}_{\mathrm{s}} & \mathbf{0} & \mathbf{0} \\
\mathbf{0} & \mathbf{K}_{\mathrm{s}} & -\mathbf{K}_{\mathrm{c}} & \mathbf{0} & \mathbf{0} & -\mathbf{K}_{\mathrm{s}} & \mathbf{K}_{\mathrm{c}} & \mathbf{0} & \mathbf{0} \\
2 \mathbf{K}_{\mathrm{c}} & \mathbf{0} & \mathbf{0} & \mathbf{0} & \mathbf{0} & \mathbf{0} & \mathbf{0} & \mathbf{K}_{\mathrm{c}} & \mathbf{K}_{\mathrm{s}} \\
2 \mathbf{K}_{\mathrm{s}} & \mathbf{0} & \mathbf{0} & \mathbf{0} & \mathbf{0} & \mathbf{0} & \mathbf{0} & -\mathbf{K}_{\mathrm{s}} & \mathbf{K}_{\mathrm{c}} \\
\mathbf{0} & \mathbf{K}_{\mathrm{c}} & -\mathbf{K}_{\mathrm{s}} & \mathbf{0} & \mathbf{0} & \mathbf{0} & \mathbf{0} & \mathbf{0} & \mathbf{0} \\
\mathbf{0} & \mathbf{K}_{\mathrm{s}} & \mathbf{K}_{\mathrm{c}} & \mathbf{0} & \mathbf{0} & \mathbf{0} & \mathbf{0} & \mathbf{0} & \mathbf{0} \\
\mathbf{0} & \mathbf{0} & \mathbf{0} & \mathbf{K}_{\mathrm{c}} & -\mathbf{K}_{\mathrm{s}} & \mathbf{0} & \mathbf{0} & \mathbf{0} & \mathbf{0} \\
\mathbf{0} & \mathbf{0} & \mathbf{0} & \mathbf{K}_{\mathrm{s}} & \mathbf{K}_{\mathrm{c}} & \mathbf{0} & \mathbf{0} & \mathbf{0} & \mathbf{0}
\end{array}\right]
\end{aligned}
$$

where

$$
\lambda_{\mathbf{h}}=\left[\begin{array}{cc}
\mathbf{K}_{\mathrm{sh}}(\tau)+\mathbf{K}_{\mathrm{sup}}+\mathbf{K}_{\mathbf{f}}^{\mathbf{c}}-h^{2} \omega^{2} \mathbf{M} & h \omega(\mathbf{C}+\omega \mathbf{G}) \\
-h \omega(\mathbf{C}+\omega \mathbf{G}) & \mathbf{K}_{\mathrm{sh}}(\tau)+\mathbf{K}_{\mathrm{sup}}+\mathbf{K}_{\mathbf{f}}^{\mathbf{c}}-h^{2} \omega^{2} \mathbf{M}
\end{array}\right]
$$

for $h=1,2,3$ and 4 .

Moreover $\lambda_{\mathbf{h}}$ may be expanded in terms of $\omega$ :

$$
\begin{aligned}
\lambda_{\mathbf{h}} & =-\omega^{2}\left[\begin{array}{cc}
h^{2} \mathbf{M} & -h \mathbf{G} \\
h \mathbf{G} & h^{2} \mathbf{M}
\end{array}\right]+\omega\left[\begin{array}{cc}
\mathbf{0} & h \mathbf{C} \\
-h \mathbf{C} & \mathbf{0}
\end{array}\right]+\left[\begin{array}{cc}
\mathbf{K}_{\mathrm{sh}}(\tau)+\mathbf{K}_{\mathrm{sup}}+\mathbf{K}_{\mathbf{f}}^{\mathbf{c}} & \mathbf{0} \\
\mathbf{0} & \mathbf{K}_{\mathrm{sh}}(\tau)+\mathbf{K}_{\mathrm{Sup}}+\mathbf{K}_{\mathbf{f}}^{\mathbf{c}}
\end{array}\right] \\
& =-\omega^{2} \lambda_{\mathbf{2 h}}+\omega \lambda_{\mathbf{1 h}}+\lambda_{\mathbf{o h}}(\tau)
\end{aligned}
$$

$\lambda_{\mathbf{0 h}}$ is a random matrix. According to equation (7) $\lambda_{\mathbf{0 h}}$ may be expanded in a deterministic part and 
a random part:

$$
\begin{aligned}
\lambda_{\mathbf{0 h}, \mathbf{d e t}} & =\left[\begin{array}{cc}
\overline{\mathbf{K}}_{\mathrm{sh}}+\mathbf{K}_{\mathrm{Sup}}+\mathbf{K}_{\mathbf{f}}^{\mathbf{c}} & \mathbf{0} \\
\mathbf{0} & \overline{\mathbf{K}}_{\mathrm{sh}}+\mathbf{K}_{\mathrm{sup}}+\mathbf{K}_{\mathbf{f}}^{\mathbf{c}}
\end{array}\right] \\
\lambda_{\mathbf{O h}, \text { rand }} & =\left[\begin{array}{cc}
\overline{\mathbf{K}}_{\mathrm{sh}} & \mathbf{0} \\
\mathbf{0} & \overline{\mathbf{K}}_{\mathrm{sh}}
\end{array}\right] \\
\lambda_{\mathbf{0 h}} & =\lambda_{\mathbf{0 h}, \mathbf{d e t}}+\delta_{E} \xi \lambda_{\mathbf{0 h}, \mathbf{r a n d}}
\end{aligned}
$$

Then matrices $\boldsymbol{\Lambda}_{\mathbf{M}}, \boldsymbol{\Lambda}_{\mathrm{C}}, \boldsymbol{\Lambda}_{\mathbf{0}}, \boldsymbol{\Lambda}_{\mathbf{0}, \text { det }}, \boldsymbol{\Lambda}_{\mathbf{0} \text {,rand }}$ are defined:

$$
\begin{aligned}
& \operatorname{diag}\left(\boldsymbol{\Lambda}_{\mathbf{M}}\right)=\left(\begin{array}{llll}
\mathbf{0} & \lambda_{\mathbf{2 1}} & \cdots & \lambda_{\mathbf{2 4}}
\end{array}\right) \\
& \operatorname{diag}\left(\boldsymbol{\Lambda}_{\mathbf{C}}\right)=\left(\begin{array}{llll}
\mathbf{0} & \lambda_{\mathbf{1 1}} & \cdots & \lambda_{\mathbf{1 4}}
\end{array}\right) \\
& \operatorname{diag}\left(\boldsymbol{\Lambda}_{\mathbf{0}, \mathbf{d e t}}\right)=\left(\overline{\mathbf{K}}_{\mathrm{sh}}+\mathbf{K}_{\mathrm{sup}}+\mathbf{K}_{\mathbf{f}}^{\mathbf{c}} \quad \lambda_{\mathbf{0 1 , \text { det }}} \cdots \lambda_{\mathbf{0 4 , \text { det }}}\right) \\
& \operatorname{diag}\left(\boldsymbol{\Lambda}_{\mathbf{0}, \text { rand }}\right)=\left(\begin{array}{llll}
\overline{\mathbf{K}}_{\text {sh }} & \lambda_{\mathbf{0 1 , \text { rand }}} \cdots \lambda_{\mathbf{0 4}, \text { rand }}
\end{array}\right) \\
& \boldsymbol{\Lambda}_{\mathbf{0}}=\boldsymbol{\Lambda}_{\mathbf{0}, \mathrm{det}}+\delta_{E} \xi \boldsymbol{\Lambda}_{\mathbf{0}, \text { rand }}
\end{aligned}
$$

Thus, similarly $\boldsymbol{\Lambda}$ may be expanded in terms of $\omega$ and a deterministic part and a random part may be identified:

$$
\boldsymbol{\Lambda}=-\omega^{2} \boldsymbol{\Lambda}_{\mathbf{M}}+\omega \boldsymbol{\Lambda}_{\mathbf{C}}+\boldsymbol{\Lambda}_{\mathrm{b}}+\boldsymbol{\Lambda}_{\mathbf{0}}=-\omega^{2} \boldsymbol{\Lambda}_{\mathbf{M}}+\omega \boldsymbol{\Lambda}_{\mathbf{C}}+\boldsymbol{\Lambda}_{\mathbf{K}, \mathrm{det}}+\delta_{E} \xi \overline{\boldsymbol{\Lambda}}_{\mathbf{K}, \mathrm{rand}}
$$

where $\Lambda_{\mathbf{K}, \text { det }}=\Lambda_{\mathrm{b}}+\Lambda_{\mathbf{0}, \text { det }}$ and $\bar{\Lambda}_{\mathbf{K}, \text { rand }}=\Lambda_{\mathbf{0 , \text { rand }}}$.

Then equation (9) may be rewritten

$$
\left(-\omega^{2} \Lambda_{\mathbf{M}}+\omega \Lambda_{\mathbf{C}}+\Lambda_{\mathbf{K}, \operatorname{det}}+\delta_{E} \xi \bar{\Lambda}_{\mathbf{K}, \text { rand }}\right) \boldsymbol{\Theta}(\tau)=\boldsymbol{\Gamma}
$$

\subsection{Stochastic response}

Response vector $\Theta$ is expanded in terms of polynomials $\Psi_{j}$ whose variables are a set of mutually independent standard normal deviates [5]. In our case, polynomial chaos $\Psi_{j}$ is a Hermite polynomial and depends on random variable $\xi(\tau)$.

So the response can be expanded on the Polynomial Chaos basis such that

$$
\boldsymbol{\Theta}=\sum_{j=0}^{\infty} \mathbf{Y}_{\mathbf{j}} \Psi_{j}(\xi(\tau))
$$

where the elements of vectors $\mathbf{Y}_{\mathbf{j}}\left(\mathbf{Y}_{\mathbf{j}} \in \mathbb{R}^{9 n}\right.$ where $n$ is the number of dof of the system, ie $n$ is the size of $\mathbf{x}$ ) are the coefficients of the expansion. In the following $\xi(\tau)$ will be written $\xi$ for the sake of simplicity.

For the numerical study this expression has to be truncated to a finite number of terms. By defining $P+1$ as the total number of polynomial chaoses used in the truncated expansion, the following relation 
can be determined: $P+1=(l+r) ! /(l ! r !)$ where $r$ is the number of random variables and $l$ is the chaos order; due to the fact that $r=1$ in the present study, we have $P=l$. Then, an approximation of $\boldsymbol{\Theta}$ is obtained as

$$
\Theta^{\mathbf{P}}=\sum_{j=0}^{P} \mathbf{Y}_{\mathbf{j}} \Psi_{j}(\xi)
$$

For the sake of simplicity, exponent $\mathbf{P}$ is dropped in the following.

Then, a two steps procedure based on an intrusive method will be applied in order to solve the problem: first, introduction of the truncated expansions (29) into model equations (27) will be performed. Second, the PC coefficients of equation (29) have to be determined via a new set of equations. The following part of the paper describes this intrusive technique for the determination of PC coefficients.

Substituting relation (29) in equation (27), one obtains:

$$
\sum_{j=0}^{P} \Psi_{j}(\xi)\left(-\omega^{2} \boldsymbol{\Lambda}_{\mathbf{M}}+\omega \boldsymbol{\Lambda}_{\mathbf{C}}+\boldsymbol{\Lambda}_{\mathbf{0}, \text { det }}+\delta_{E} \xi \overline{\boldsymbol{\Lambda}}_{\mathbf{0}, \text { rand }}\right) \mathbf{Y}_{\mathbf{j}}=\boldsymbol{\Gamma}
$$

By multiplying equation (30) by $\Psi_{k}(\xi)$ and using the Hermite polynomial properties, the following equations are obtained:

$$
\forall k=0 \cdots P, \quad<0, k, k>\left(-\omega^{2} \boldsymbol{\Lambda}_{\mathbf{M}}+\omega \boldsymbol{\Lambda}_{\mathbf{C}}+\boldsymbol{\Lambda}_{\mathbf{0}, \mathbf{d e t}}\right) \mathbf{Y}_{\mathbf{k}}+\sum_{j=0}^{P} \delta_{E}<1, k, j>\overline{\boldsymbol{\Lambda}}_{\mathbf{0}, \text { rand }} \mathbf{Y}_{\mathbf{j}}=\delta_{0 k} \boldsymbol{\Gamma}
$$

where $\delta_{0 k}$ is the Kronecker delta and

$$
<k, i, j>=\int_{-\infty}^{+\infty} \xi^{k} \Psi_{i}(\xi) \Psi_{j}(\xi) \frac{e^{-\xi^{2} / 2}}{\sqrt{2 \pi}} \mathrm{d} \xi
$$

The following relations may be easily derived:

$$
\begin{aligned}
& <0, i, j>=\int_{-\infty}^{+\infty} \Psi_{i}(\xi) \Psi_{j}(\xi) \frac{e^{-\xi^{2} / 2}}{\sqrt{2 \pi}} \mathrm{d} \xi=j ! \delta_{i j} \\
& <1, i, j>=\int_{-\infty}^{+\infty} \xi \Psi_{i}(\xi) \Psi_{j}(\xi) \frac{e^{-\xi^{2} / 2}}{\sqrt{2 \pi}} \mathrm{d} \xi=\delta_{j i+1} j !+\delta_{j i-1}(j+1) !
\end{aligned}
$$

Define

$$
\begin{aligned}
& \mathbf{M o m}^{\mathbf{0}} / \mathbf{M o m}_{i j}^{\mathbf{0}}=<0, i, j>, \mathbf{M o m}^{\mathbf{0}} \in \mathbb{R}^{(P+1) \times(P+1)} \\
& \mathbf{M o m}^{\mathbf{1}} / \mathbf{M o m}^{\mathbf{1}}{ }_{i j}=<1, i, j>, \mathbf{M o m}^{\mathbf{1}} \in \mathbb{R}^{(P+1) \times(P+1)} \\
& \widetilde{\boldsymbol{\Lambda}_{\mathbf{M}}}=\mathbf{M o m}^{\mathbf{0}} \otimes \boldsymbol{\Lambda}_{\mathbf{M}} \in \mathbb{R}^{9 n(P+1) \times 9 n(P+1)} \\
& \widetilde{\boldsymbol{\Lambda}_{\mathbf{C}}}=\mathbf{M o m}^{\mathbf{0}} \otimes \boldsymbol{\Lambda}_{\mathbf{C}} \in \mathbb{R}^{9 n(P+1) \times 9 n(P+1)} \\
& \widetilde{\boldsymbol{\Lambda}_{\mathbf{K}}}=\mathbf{M o m}^{\mathbf{0}} \otimes \boldsymbol{\Lambda}_{\mathbf{0}, \mathbf{d e t}}+\delta_{E} \mathbf{M o m}^{\mathbf{1}} \otimes \boldsymbol{\Lambda}_{\mathbf{0}, \text { rand }} \in \mathbb{R}^{9 n(P+1) \times 9 n(P+1)} \\
& \mathbf{Y}=\left[\mathbf{Y}_{\mathbf{0}}^{T} \cdots \mathbf{Y}_{\mathbf{P}^{T}}\right]^{T} \in \mathbb{R}^{9 n(P+1)} \\
& \widetilde{\boldsymbol{\Gamma}}=\left[\begin{array}{lllll}
\boldsymbol{\Gamma}^{T} & 0 & 0 & \cdots & 0
\end{array}\right]^{T} \in \mathbb{R}^{9 n(P+1)}
\end{aligned}
$$


where $\otimes$ denotes the Kronecker product. Then the components of the PC expansion satisfy the following equation:

$$
\left(-\omega^{2} \widetilde{\boldsymbol{\Lambda}_{\mathrm{M}}}+\omega \widetilde{\boldsymbol{\Lambda}_{\mathrm{C}}}+\widetilde{\boldsymbol{\Lambda}_{\mathbf{K}}}\right) \mathbf{Y}=\widetilde{\boldsymbol{\Gamma}}
$$

System of equations (42) may be solved directly. However it involves the calculation of $i$ ! that may be very quickly beyond the precision of the computers (e.g. $20 ! \simeq 2.410^{18}$ ). Moreover, its size rises with the PC order and some difficulties to solve the system may arise: some trouble for $P$ greater to 30 has been observed for some rotating speeds in this present study. Thus it becomes impracticable to carry out calculations of stochastic vibration responses for rotating speeds of interest. However, these issues may be overcome quite easily by writing the system in detail. Indeed system of equations (42) gathers $P+1$ matrix equations:

$$
\begin{aligned}
& 0 ! \mathbf{A} \mathbf{Y}_{\mathbf{0}}+1 ! \delta_{E} \overline{\boldsymbol{\Lambda}}_{\mathbf{0}, \text { rand }} \mathbf{Y}_{\mathbf{1}}=\boldsymbol{\Gamma} \\
& 1 \text { ! } \mathbf{A} \mathbf{Y}_{\mathbf{1}}+1 ! \delta_{E} \overline{\boldsymbol{\Lambda}}_{\mathbf{0}, \text { rand }} \mathbf{Y}_{\mathbf{0}}+2 \text { ! } \delta_{E} \overline{\boldsymbol{\Lambda}}_{\mathbf{0}, \text { rand }} \mathbf{Y}_{\mathbf{2}}=\mathbf{0} \\
& \cdots=\ldots \\
& i ! \mathbf{A} \mathbf{Y}_{\mathbf{i}}+i ! \delta_{E} \overline{\boldsymbol{\Lambda}}_{\mathbf{0}, \text { rand }} \mathbf{Y}_{\mathbf{i}-\mathbf{1}}+(i+1) ! \delta_{E} \overline{\boldsymbol{\Lambda}}_{\mathbf{0}, \text { rand }} \mathbf{Y}_{\mathbf{i}+\mathbf{1}}=\mathbf{0} \\
& \cdots=\ldots \\
& (P-1) ! \mathbf{A} \mathbf{Y}_{\mathbf{P}-\mathbf{1}}+(P-1) ! \delta_{E} \overline{\boldsymbol{\Lambda}}_{\mathbf{0} \text {,rand }} \mathbf{Y}_{\mathbf{P}-\mathbf{2}}+P ! \delta_{E} \overline{\boldsymbol{\Lambda}}_{\mathbf{0} \text {,rand }} \mathbf{Y}_{\mathbf{P}}=\mathbf{0} \\
& P ! \mathbf{A} \mathbf{Y}_{\mathbf{P}}+P ! \delta_{E} \overline{\mathbf{\Lambda}}_{\mathbf{0}, \text { rand }} \mathbf{Y}_{\mathbf{P}-\mathbf{1}}=\mathbf{0}
\end{aligned}
$$

with $\mathbf{A}=-\omega^{2} \boldsymbol{\Lambda}_{\mathbf{M}}+\omega \boldsymbol{\Lambda}_{\mathbf{C}}+\boldsymbol{\Lambda}_{\mathbf{0}, \mathbf{d e t}}$.

First, we can note that each equation $i+1$ may be simplified by dividing this equation by $i$ !. Then no factorial is involved in finding $\mathbf{Y}_{\mathbf{k}}$ : this removes the issue to determine $i$ ! for large $i$.

Second, system of equations (42) may be recursively solved by using the following relations:

$$
\begin{aligned}
\forall i>1, \quad \mathbf{Y}_{\mathbf{i}} & =\mathbf{D}_{\mathbf{i}}^{-1}\left(-\delta_{E} \overline{\boldsymbol{\Lambda}}_{\mathbf{0}, \text { rand }}\right) \mathbf{Y}_{\mathbf{i}-\mathbf{1}} \\
\mathbf{Y}_{\mathbf{0}} & =\left(\mathbf{A}+\delta_{E} \overline{\boldsymbol{\Lambda}}_{\mathbf{0}, \text { rand }}\right)^{-1} \mathbf{\Gamma}
\end{aligned}
$$

where matrix sequence $\mathbf{D}_{\mathbf{i}}$ verifies the recursive relation:

$$
\begin{aligned}
\mathbf{D}_{\mathbf{i}} & =\mathbf{A}-(i+1) \delta_{E}^{2} \overline{\boldsymbol{\Lambda}}_{\mathbf{0}, \text { rand }} \mathbf{D}_{\mathbf{i}+\mathbf{1}}{ }^{-1} \overline{\boldsymbol{\Lambda}}_{\mathbf{0}, \text { rand }} \\
\mathbf{D}_{\mathbf{P}} & =\mathbf{A}
\end{aligned}
$$

Using equations (48) to (51), $P+1$ systems of size $9 n \times 9 n$ have to be solved, instead of one system of size $9 n(P+1) \times 9 n(P+1)$. In that way, it is possible to solve the problem event for large $P$, as it will be seen in the next sections. 
It is of interest to remark that this procedure involves a numerical cost similar to the numerical cost of a direct Monte-Carlo simulation that uses $(P+1)$ samples: indeed the latter needs to solve $P+1$ times system of equations (9). Accordingly, the PC expansion will be an efficient method compared to the Monte Carlo Simulations (MCS) only if the PC order is much lower that the number of samples to achieve the same accuracy with both calculations.

Due to the fact that Monte Carlo simulation has poor convergence properties and requires much more simulations (10,000 or 100,000 simulations are usual) than PC expansion, this recursive methodology is more effective than the direct Monte-Carlo simulation.

\section{Numerical results}

First of all, the deterministic dynamic response of the rotor will be investigated. The objective of the first part of this section is to have an initial understanding of the behavior of the deterministic system. This will then allow us a better understanding of the results obtained by taking into account uncertainty, which will be achieved in the second part of this section.

\subsection{Deterministic dynamic response of the rotor}

The vertical and horizontal displacements of the rotor system (for the first, second, third and fourth orders of the super harmonics for the steady-state response) are plotted in Figure 2: in this figure and in the next ones, the $1 \times$ (respectively $2 \times, 3 \times$ and, $4 \times$ ) amplitude is a blue (respectively red, magenta and, green) line. The range of rotation speed is [50;350] rpm, and the rotation speed step is $0.5 \mathrm{rpm}$. These results clearly demonstrate that the presence of an asymmetric coupling results in the appearance of super harmonics components. The $2 \times$ harmonics (i.e. the harmonics of order 2) are observed in the horizontal and vertical directions at one-half of the first backward and forward critical speeds (as shown in Figures 2(a) and (b) around $[150 ; 152] \mathrm{rpm}$ ). Then, the $3 \times$ and $4 \times$ harmonics in the horizontal and vertical directions are also present at one-third and one-fourth of the first backward and forward critical speeds (at [100; 102] rpm and [75; 76] rpm respectively). It can also be noted that there are visible $3 \times$ harmonics $(4 \times$ harmonics respectively) at the first backward and forward critical speeds (at the one-half of first backward and forward critical speeds respectively) due to the coupling terms of $\boldsymbol{\Lambda}_{b}$. Finally the $2 \times$ amplitude peaks $(3 \times$ and $4 \times$, respectively) around $[210 ; 220] \mathrm{rpm}$ ([140;150] rpm and [100; 110] rpm, respectively) corresponds to the crossing of one-half, one-third and one-fourth of the second forward and backward critical speeds.

[Figure 2 about here.]

\subsection{Requested quantities and Monte-Carlo simulation}

Equation (42) provides $\mathbf{Y}$, which in turn gives $\boldsymbol{\Theta}$ as shown by equation (29). So it is possible to derive the mean and the standard deviation of each component of $\Theta$ by running a Monte-Carlo 
simulation on the random variable $\xi$ :

$$
\begin{aligned}
& \overline{\mathbf{A}_{\mathbf{0}}}=\frac{1}{n_{M C S}} \sum_{k=1}^{n_{M C S}} \sum_{j=0}^{P} \mathbf{Y}_{\mathbf{0}_{j}} \Psi_{j}\left(\xi_{k}\right) \\
& \forall h \in\{1, \cdots, 4\}, \overline{\mathbf{A}_{\mathbf{h}}}=\frac{1}{n_{M C S}} \sum_{k=1}^{n_{M C S}} \sum_{j=0}^{P} \mathbf{Y}_{\mathbf{2 h}-\mathbf{1} j} \Psi_{j}\left(\xi_{k}\right) \\
& \forall h \in\{1, \cdots, 4\}, \quad \overline{\mathbf{B}_{\mathbf{h}}}=\frac{1}{n_{M C S}} \sum_{k=1}^{n_{M C S}} \sum_{j=0}^{P} \mathbf{Y}_{\mathbf{2}} \mathbf{h}_{j} \Psi_{j}\left(\xi_{k}\right) \\
& \overline{\overline{\mathbf{A}_{\mathbf{0}}}}=\sqrt{\frac{1}{n_{M C S}}\left\{\sum_{k=1}^{n_{M C S}}\left(\sum_{j=0}^{P} \mathbf{Y}_{\mathbf{0}_{j}} \Psi_{j}\left(\xi_{k}\right)\right)^{2}\right\}-{\overline{\mathbf{A}_{\mathbf{h}}}}^{2}} \\
& \forall h \in\{1, \cdots, 4\}, \overline{\overline{\mathbf{A}_{\mathbf{h}}}}=\sqrt{\frac{1}{n_{M C S}}\left\{\sum_{k=1}^{n_{M C S}}\left(\sum_{j=0}^{P} \mathbf{Y}_{\mathbf{2 h}-\mathbf{1}_{j}} \Psi_{j}\left(\xi_{k}\right)\right)^{2}\right\}-\overline{\mathbf{A}}_{\mathbf{h}}{ }^{2}} \\
& \forall h \in\{1, \cdots, 4\}, \overline{\overline{\mathbf{B}_{\mathbf{h}}}}=\sqrt{\frac{1}{n_{M C S}}\left\{\sum_{k=1}^{n_{M C S}}\left(\sum_{j=0}^{P} \mathbf{Y}_{\mathbf{2 h}} \Psi_{j}\left(\xi_{k}\right)\right)^{2}\right\}-\overline{\mathbf{B}}_{\mathbf{h}}^{2}}
\end{aligned}
$$

where ${ }^{-}\left(\right.$respectively ${ }^{\bar{*}}$ ) refers to the mean (respectively to the standard deviation). The vectors $\mathbf{Y}_{\mathbf{0} j}$, $\mathbf{Y}_{\mathbf{2 h}-\mathbf{1} j}$ and $\mathbf{Y}_{\mathbf{2 h}}$ correspond to the partitioning of the vector $\mathbf{Y}$ for the chaos order $j(j=0,1, \ldots, P)$. $\xi_{k}$ is a realization of random variable $\xi ; n_{M C S}$ is the number of draws of the MCS.

Then the mean and the standard deviation of response $\mathbf{x}_{\mathbf{h}}$ of order $h>0$ (for $h=1,2,3$ and 4 ) are defined as

$$
\begin{aligned}
& \overline{\mathbf{x}_{\mathbf{h}}}(\omega)=\sqrt{{\overline{\mathbf{A}_{\mathbf{h}}}}^{2}+{\overline{\mathbf{B}_{\mathbf{h}}}}^{2}} \\
& \overline{\overline{\mathbf{x}_{\mathbf{h}}}}(\omega)=\sqrt{{\overline{\overline{\mathbf{A}_{\mathbf{h}}}}}^{2}+{\overline{\mathbf{B}_{\mathbf{h}}}}^{2}}
\end{aligned}
$$

A Monte-Carlo Simulation was carried out. It took around 400 minutes to perform a 10000 draws simulation. The results are given in Figures 3. These results will serve as a reference solution for the next section of the paper. It may be recalled that the Monte Carlo Simulation has poor convergence properties and required a great number of samples (10,000 simulations are usual).

[Figure 3 about here.]

\subsection{Influence of the $P C$ expansion}

Figure 4 and 5 show the mean and standard deviation of rotor response at mid-span when using a PC order 2 and 3 respectively. Comparing these results with the results obtained using MCS, it is obvious that the mean and standard deviation are poorly evaluated around critical speeds. PC expansions with such low orders successively overestimate or underestimate strongly these first two centered moments, giving birth to resonance-like peaks which may be interpreted as spurious critical 
speeds. Indeed, whenever $\omega$ is close to an eigenvalue of $\left(\widetilde{\boldsymbol{\Lambda}_{\mathbf{K}}}, \widetilde{\boldsymbol{\Lambda}_{\mathbf{M}}}\right)$, , the solution $\mathbf{Y}$ of equation (42) exhibits large components and creates these spurious resonance peaks. By increasing the PC order, the spurious critical speeds merge and the curves smooth, as shown in Figures 6 and 7, when $P=30$ and $P=200$ respectively. This simulation with $P=30$ lasted 10 times less than the MCS.

Figure 8 explains why a high PC order is required for some rotation speeds. Indeed, the peaks of the $1 \times$ and $3 \times$ responses in function of $\xi$ can not be well estimated with low degree polynomials: that explains why, for these rotation speeds a very high number of PC will be required to estimate the first two moments. It can also be observed that peaks are visible only for the $1 \times$ and $3 \times$ components due to the coupling between the $n \times$ components (see the expression of $\boldsymbol{\Lambda}_{\mathbf{b}}$, equation (14)).

However the shape of the $1 \times$ and $3 \times$ responses are not easily represented by polynomials: that explains why, for this rotation speed a high number of PC is required to estimate the first two moments.

This difficulty to properly approach the responce near critical speeds may be related to the work in [8] which focuses on a single-dof linear oscillator and exhibits a multimodal stochastic response hard to represent next to the resonance frequency

[Figure 4 about here.]

[Figure 5 about here.]

[Figure 6 about here.]

[Figure 7 about here.]

[Figure 8 about here.]

Figure 7 seems to show that the results obtained with $P=200$ does not improve so much the results obtained with $P=30$ : however, this is only due to the logarithmic scale. Indeed, consider the first harmonic for the mean vertical displacement critical speed at $300.3 \mathrm{rpm}, \mathbf{x}_{v, 1 x}(P)$; the evolution of this variable is plotted in Figure 9 on a linear scale: the strong dependency on the PC order is striking. First, it can be seen that the response for $P=30$ is 2.2 time larger than the response for $P=200$. Some extra calculations showed that the latter is still 1.5 larger than the response for $P=2000$. So the convergence is not reached even for $P=200$ : around the critical speeds a very high $\mathrm{PC}$ order is required. Second, there is a strong dependency on the parity of $P$. Indeed, considering the sequences $\mathbf{x}_{v, 1 x}(2 P)$ and $\mathbf{x}_{v, 1 x}(2 P+1)$, the first one is strictly monotonically decreasing whereas the second one is strictly monotonically increasing. So, if the ratio of two successive terms of each sequence is around 1 , the ratio of $\mathbf{x}_{v, 1 x}(2 P)$ to $\mathbf{x}_{v, 1 x}(2 P+1)$ may be large: for instance $\mathbf{x}_{v, 1 x}(30) / \mathbf{x}_{v, 1 x}(32) \simeq 1.03$ and $\mathbf{x}_{v, 1 x}(30) / \mathbf{x}_{v, 1 x}(31) \simeq 12.4$. Figure 10 shows that $2 P$ should be around 1000 to obtained a ratio of 
two consecutive terms close to one, i.e. to have a relative difference $\mathcal{D}(P)$ less than $10 \%$, with

$$
\mathcal{D}(P)=\frac{\left\|\mathbf{x}_{v, 1 x}(P+1)-\mathbf{x}_{v, 1 x}(P)\right\|_{2}}{\left\|\mathbf{x}_{v, 1 x}(P)\right\|_{2}}
$$

Figure 10 and relative difference $\mathcal{D}(P)$ provide an interesting way to estimate a $\mathrm{PC}$ order that leads to a suitable accuracy: they show that PCs with order 1000 are required for the studied case around the critical speeds.

[Figure 9 about here.]

[Figure 10 about here.]

These results are particularly interesting around the critical speed at $150.2 \mathrm{rpm}$ where similar features may be observed: in particular the same ratio between the $2 \times$ mean vertical response for $P=30$ (resp. 200) to the one obtains with $P=200$ (resp. 2000) are achieved. Figures 5 to 6 shows a larger $2 \times$ mean response than the $1 \times$ one: their ratio is equal to 5.9 for $P=2$ and is still equal to 2 for $P=30$. Figure 11 shows that such a ratio is less than one only from $P=160$ and is equal to 0.92 for $P=200$. Then, contrary to the deterministic case, the $1 \times$ mean response is always greater than the $2 \times$ mean response. Also it can be noted that the mean system has not the same properties that the mean response.

As previously explained in Section 3.3, this recursive methodology is more effective than the direct Monte-Carlo simulation due to the fact that Monte Carlo simulation has poor convergence properties and required much more simulations (10,000 or 100,000 simulations are usual) than PC. Effectively with 2000 PC, the results will be more accurate than with a MCS with 2000 trials for which the convergence is not guaranteed.

[Figure 11 about here.]

\section{Conclusion}

The steady-state response of an uncertain asymmetric rotor was estimated with a PC expansion: it was compared to the one obtained with a MC simulation. The PC expansion response shows spurious oscillations around all the critical rotation speeds: the level of the oscillations decreases by rising the $\mathrm{PC}$ order that produces numerical issues. A recursive procedure allows to tackle these issues and to be able to expand the response with a high PC order. It was then shown that hundreds of PC are required to be close to the $\mathrm{MC}$ results. Likewise, the response is very sensitive to the parity of the $\mathrm{PC}$ order specially when the PC order is low: a criterion on the PC convergence was proposed by comparing the responses obtained with two consecutive PC order.

The estimation of the response with high PC order has a numerical cost. So research on that topic must focus on accelerating the PC expansion convergence in order to make it accurate and efficient. 


\section{References}

[1] Loeve, M., "Perturbation Methods". fourth ed., Springer-Verlag, $197 \%$.

[2] Benaroya, H., and Rehak, M., "Finite element methods in probabilistic structural analysis: a selective review". Applied Mechanics Reviews, 41(5), 201-213, 1988.

[3] Yamazaki, F., Shinozuka, M., and Dasgupta, G., "Neumann expansion for stochastic finite element analysis". Journal of Engineering Mechanics ASCE, 114 (8), 1335-1354, 1988.

[4] Ghanem, R., and Spanos, D., "Stochastic Finite Elements: A Spectral Approach". SpringerVerlagDover, 1991.

[5] Ghanem, R., "Stochastic finite elements with multiple random non- gaussian properties". Journal of Engineering Mechanics, 125, 26-40, 1999.

[6] Xiu, D., and Karniadakis, G.E., "Modeling uncertainty in flow simulations via generalized polynomial chaos". Journal of Computational Physics, 187, 137-167, 2003.

[7] Xiu, D., and Karniadakis, G.E., "The Wiener-Askey Polynomial Chaos for Stochastic Differential Equations". Journal on Scientific Computing, 24, 619-644, 2002.

[8] Pagnacco, E., Sarrouy, E., Sampaio, R., and Souza de Cursi, E. "Polynomial chaos for modeling multimodal dynamical systems - Investigations on a single degree of freedom system". Proceeding of XX Congress on Numerical Methods and their Applications, 2013, XXXII, 705-727.

[9] Wan, X., and Karniadakis, G.E., "An adaptive multi-element generalized polynomial chaos method for stochastic differential equations". Journal of Computational Physics, 209, 617-642, 2005.

[10] Le Meitour, J., Lucor, D., and Chassaing, J.-C. "Prediction of stochastic limit cycle oscillations using an adaptive polynomial chaos method". Journal of Aeroelasticity and Structural Dynamics, 2(1), 3-22, 2010.

[11] Sarrouy, E., Dessombz, O., and Sinou, J.J., "Stochastic study of a non-linear self-excited system with friction". European Journal of Mechanics - A/Solids, 40, 1-10, 2013.

[12] Didier, J., Faverjon, B., and Sinou, J.-J., "Analyzing the dynamic response of a rotor system under uncertain parameters by Polynomial Chaos Expansion". Journal of Vibration and Control, 18(5), 587-607, 2012.

[13] Didier, J., Sinou, J.-J., and Faverjon, B., "Study of the non-linear dynamic response of a rotor system with faults and uncertainties". Journal of Sound and Vibration, 331(3), 671-703, 2012. 
[14] Didier, J., Sinou, J.-J., and Faverjon, B., "Multi-dimensional harmonic balance with uncertainties applied to rotor dynamics". Journal of Vibration and Acoustics, 134(6), 061003, 17 pages, 2012.

[15] Sinou, J.-J., and Faverjon, B., "The vibration signature of chordal cracks in a rotor system including uncertainties". Journal of Sound and Vibration, , 331(1), 138-154, 2012.

[16] Friswell, M.I., Penny, J.E.T., Garvey, S.D. and Lees, A.W, "Dynamics of rotating machines ". Campbridge press, 2012.

[17] Ishida, Y., and Yamamoto, T., "Linear and Nonlinear Rotordynamics: A Modern Treatment with Applications". second ed., Wiley-Verlag, 2012. 


\section{List of Figures}

1 Finite-element model of the rotor with the asymmetric coupling (a) rotor system (b) asymmetric coupling . . . . . . . . . . . . . . . . . .

2 Deterministic Fourier coefficients vertical response (a), horizontal response (b), (blue= $1 \times$ response, $r e d=2 \times$ response, magenta $=3 \times$ response, green $=4 \times$ response) $\ldots \ldots$

3 Monte-Carlo Simulation; vertical response of orders 1 to 4 at the mid-span : mean (a), standard deviation (b); horizontal response of orders 1 to 4 at the mid-span : mean (c), standard deviation $(\mathrm{d}),($ blue $=1 \times$ response, red $=2 \times$ response, magenta $=3 \times$ response, green $=4 \times$ response) $\ldots \ldots \ldots \ldots \ldots \ldots$

$4 \quad \mathrm{PC}$ order $=2$; vertical response of order 1 to 4 at the mid-span : mean (a), standard deviation (b); horizontal response of order 1 to 4 at the mid-span : mean (c), standard deviation $(\mathrm{d}),($ blue $=1 \times$ response, $r e d=2 \times$ response, magenta $=3 \times$ response, green $=$ $4 \times$ response) . . . . . . . . . . . . . . . . . . . . . .

$5 \quad \mathrm{PC}$ order $=3$; vertical response of order 1 to 4 at the mid-span : mean (a), standard deviation (b); horizontal response of order 1 to 4 at the mid-span : mean (c), standard deviation $(\mathrm{d}),($ blue $=1 \times$ response, red $=2 \times$ response, magenta $=3 \times$ response, green $=$ $4 \times$ response) . . . . . . . . . . . . . . . . . . . . .

$6 \quad \mathrm{PC}$ order $=30$; vertical response of order 1 to 4 at the mid-span : mean (a), standard deviation (b); horizontal response of order 1 to 4 at the mid-span : mean (c), standard deviation $(\mathrm{d}),($ blue $=1 \times$ response, red $=2 \times$ response, magenta $=3 \times$ response, green $=$

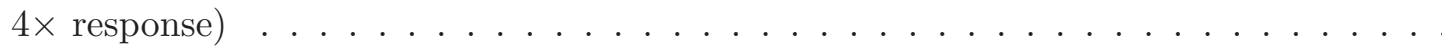

$7 \quad \mathrm{PC}$ order $=200$; vertical response of order 1 to 4 at the mid-span : mean (a), standard deviation (b); horizontal response of order 1 to 4 at the mid-span : mean (c), standard deviation $(\mathrm{d}),($ blue $=1 \times$ response, red $=2 \times$ response, magenta $=3 \times$ response, green $=$

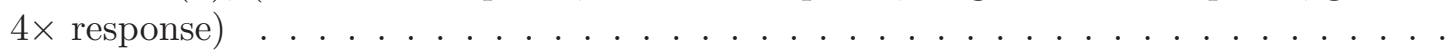

8 Distribution of the horizontal (a) and vertical (b) responses of order 1 to 4 at the midspan at $\omega=300 \mathrm{rpm},($ blue $=1 \times$ response, $\mathrm{red}=2 \times$ response, magenta $=3 \times$ response, green $=4 \times$ response $) \ldots \ldots \ldots \ldots \ldots \ldots \ldots \ldots$

$91 \times$ Mean vertical response vs. PC order at $300.3 \mathrm{rpm} \ldots \ldots \ldots \ldots \ldots$

10 Relative difference $\mathcal{D}(P)$ vs. $\mathrm{PC}$ order $\ldots \ldots \ldots \ldots \ldots \ldots$

$111 \times$ (dashed line) and $2 \times$ (solid line) mean vertical response vs. PC order at $150.2 \mathrm{rpm}$ 


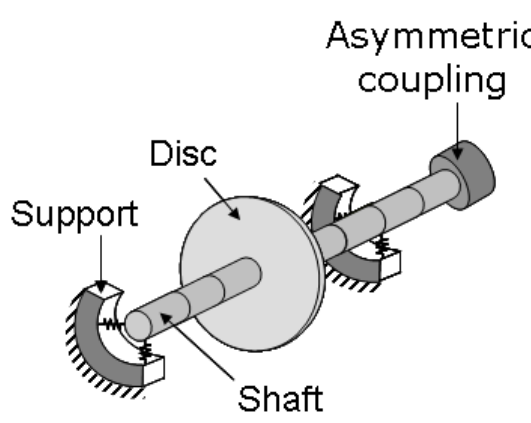

(a)

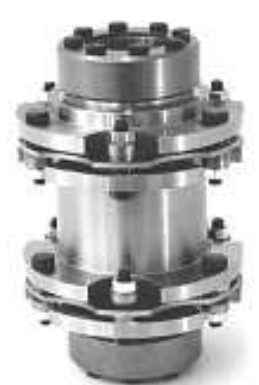

(b)

Figure 1: Finite-element model of the rotor with the asymmetric coupling (a) rotor system (b) asymmetric coupling 


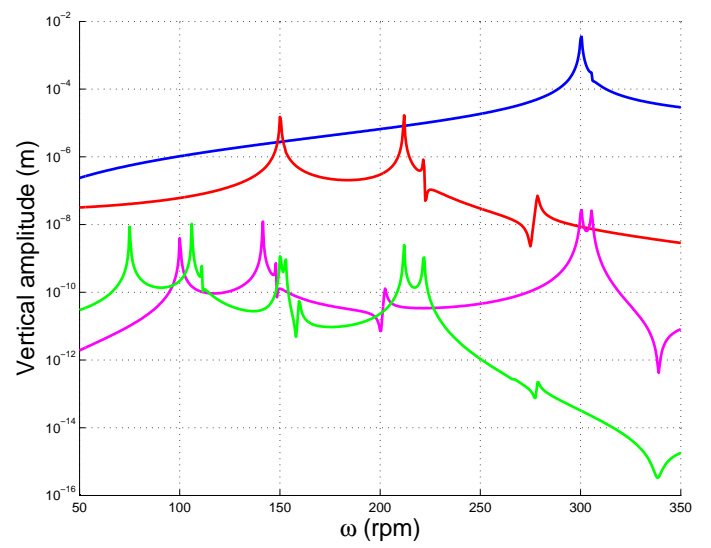

(a)

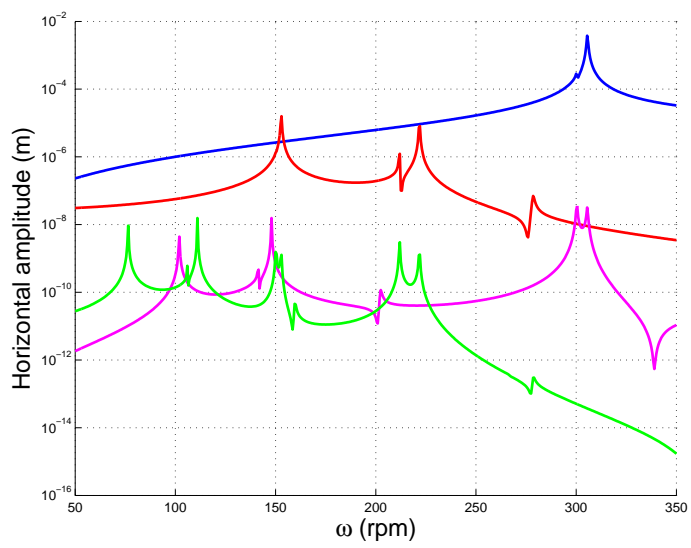

(b)

Figure 2: Deterministic Fourier coefficients vertical response (a), horizontal response (b), (blue $=1 \times$ response, red $=2 \times$ response, magenta $=3 \times$ response, green $=4 \times$ response) 


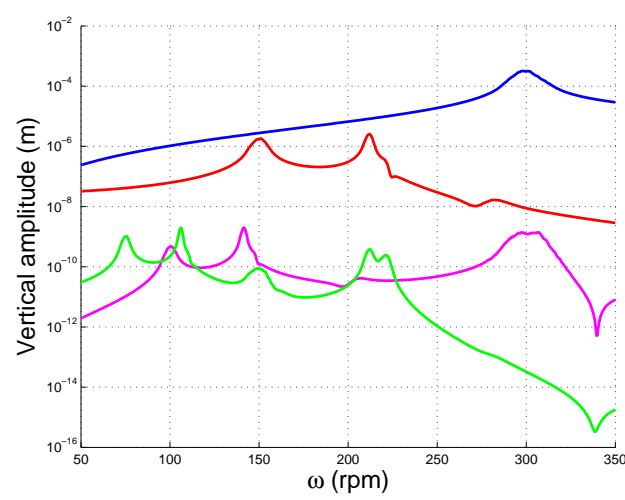

(a)

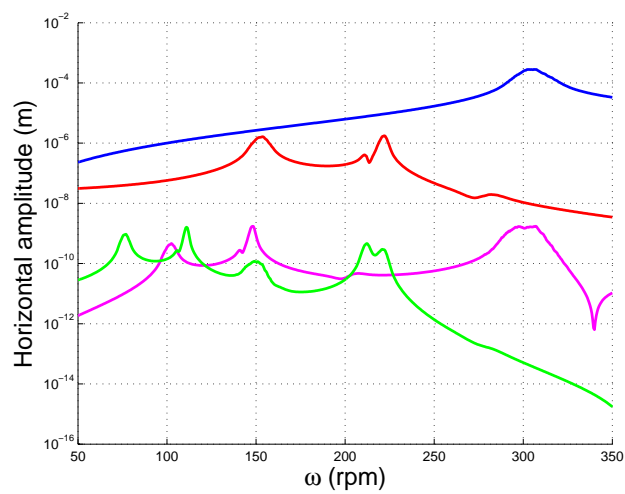

(c)

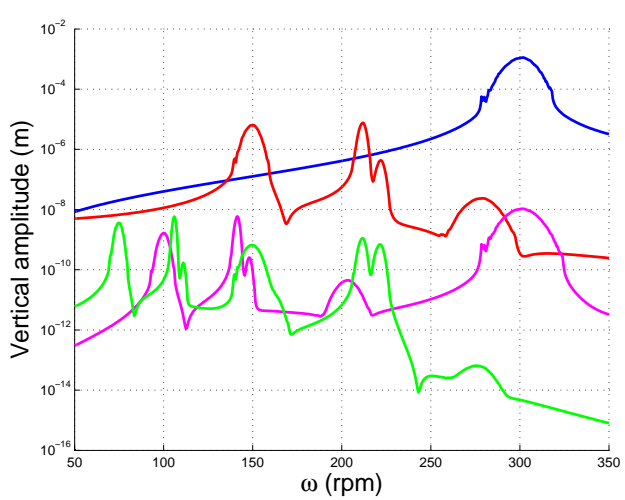

(b)

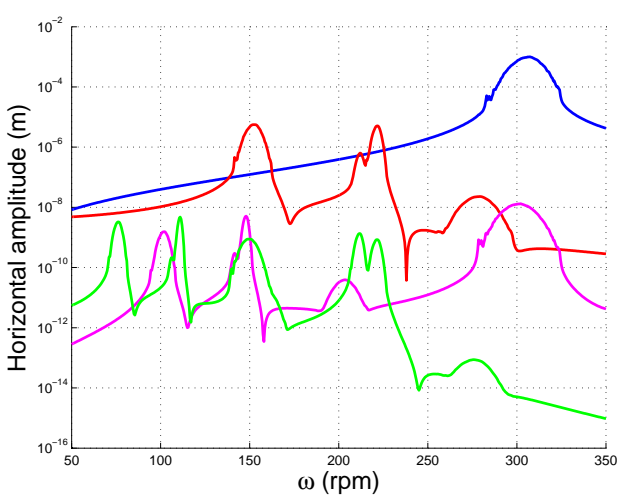

(d)

Figure 3: Monte-Carlo Simulation; vertical response of orders 1 to 4 at the mid-span : mean (a), standard deviation (b); horizontal response of orders 1 to 4 at the mid-span : mean (c), standard deviation (d), (blue $=1 \times$ response, red $=2 \times$ response, magenta $=3 \times$ response, green $=4 \times$ response). 


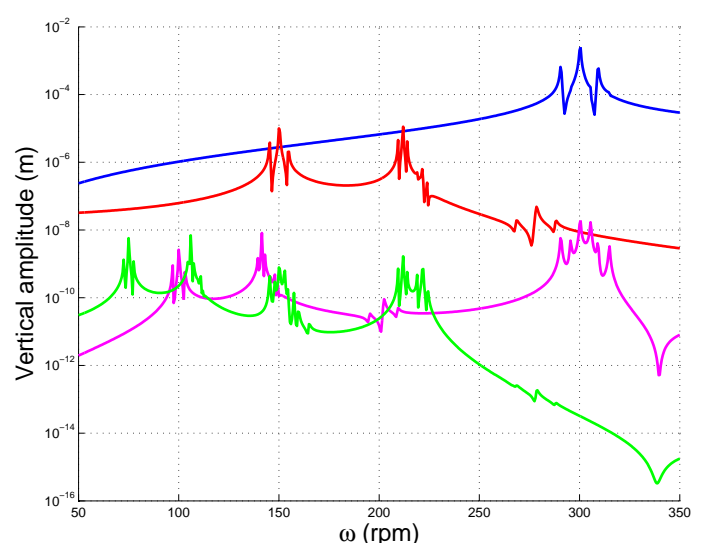

(a)

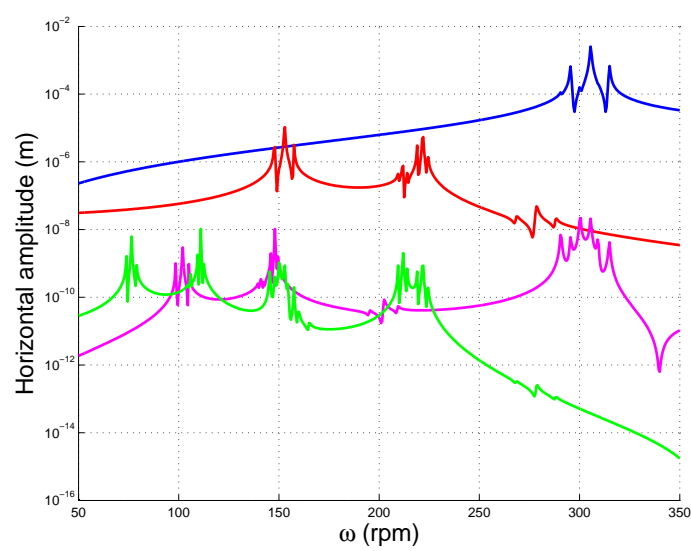

(c)

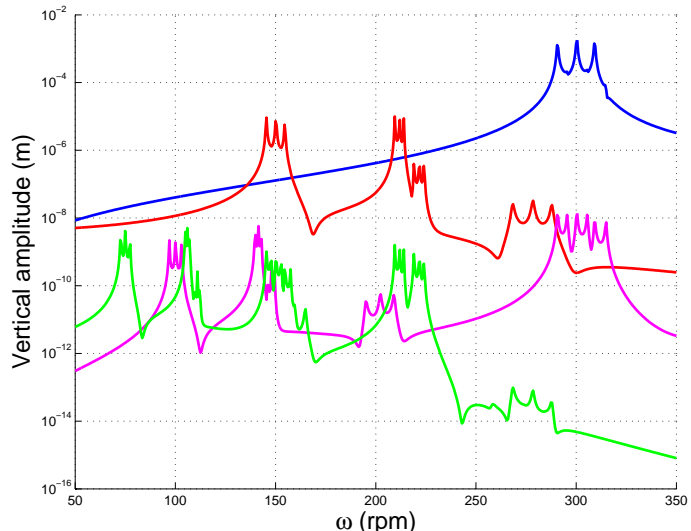

(b)

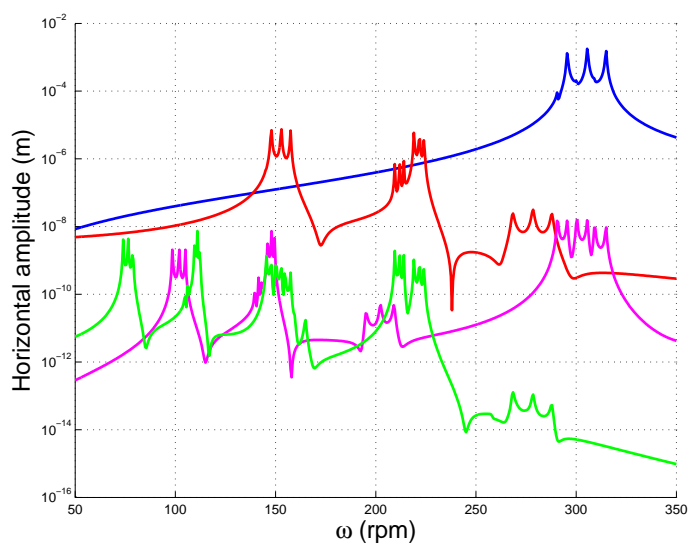

(d)

Figure 4: PC order=2; vertical response of order 1 to 4 at the mid-span : mean (a), standard deviation (b); horizontal response of order 1 to 4 at the mid-span : mean (c), standard deviation (d), (blue $=1 \times$ response, red $=2 \times$ response, magenta $=3 \times$ response, green $=4 \times$ response) . 


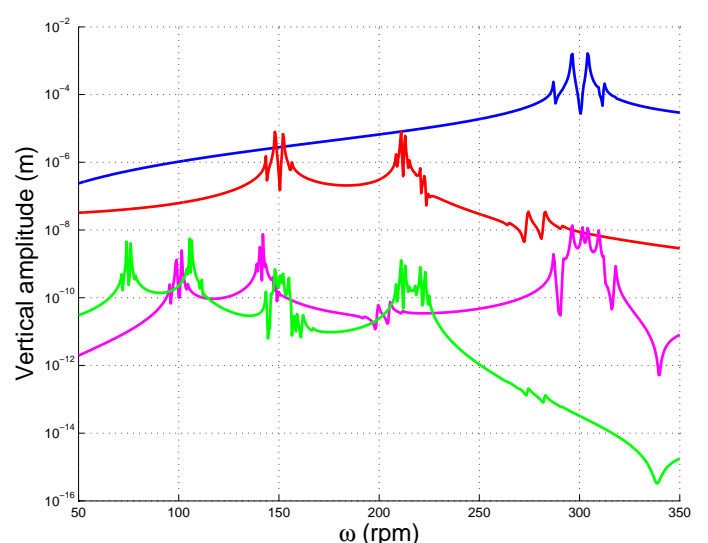

(a)

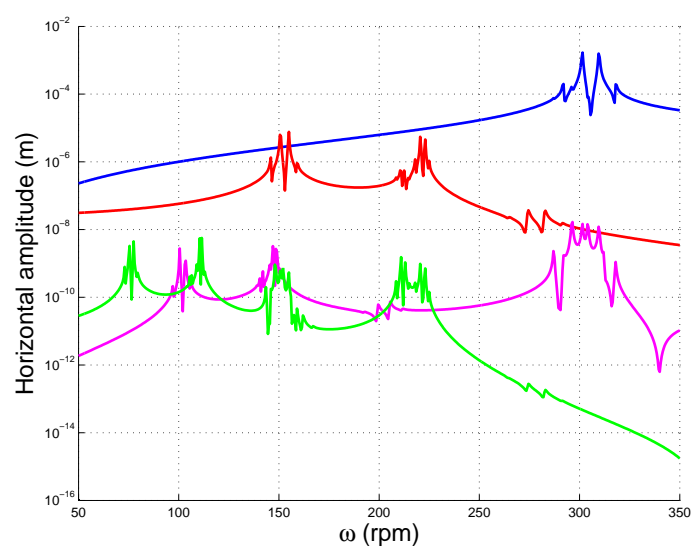

(c)

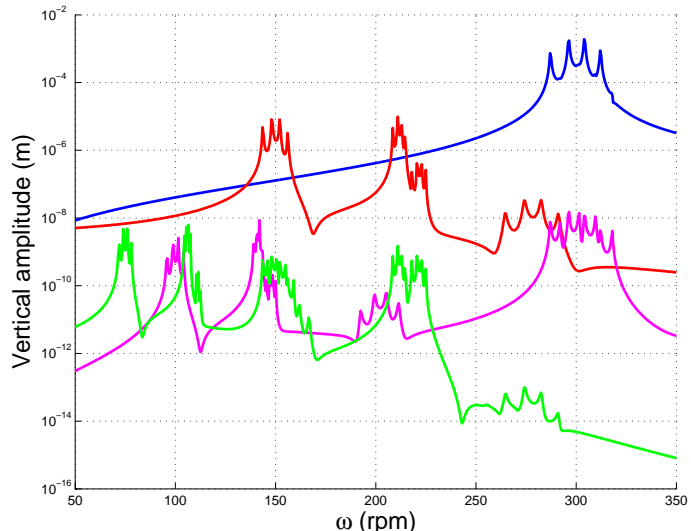

(b)

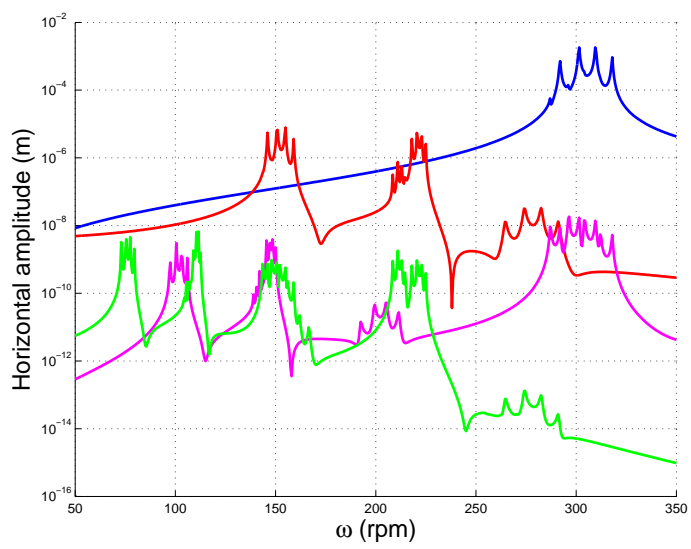

(d)

Figure 5: PC order=3; vertical response of order 1 to 4 at the mid-span : mean (a), standard deviation (b); horizontal response of order 1 to 4 at the mid-span : mean (c), standard deviation (d), (blue $=1 \times$ response, red $=2 \times$ response, magenta $=3 \times$ response, green $=4 \times$ response) . 


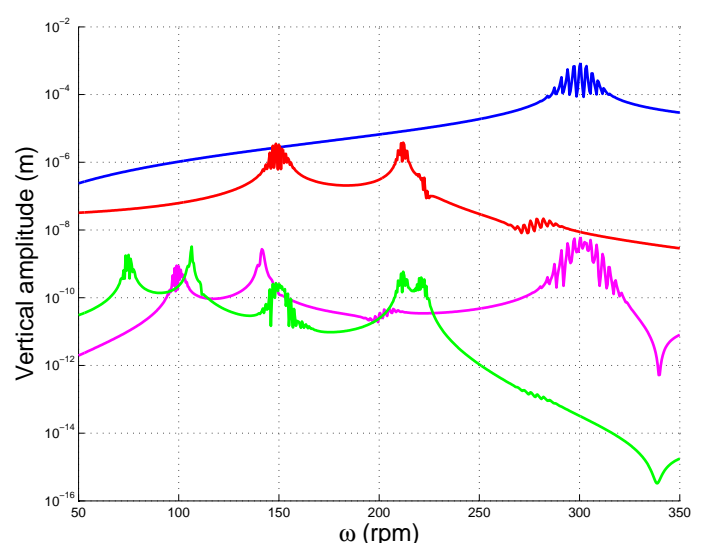

(a)

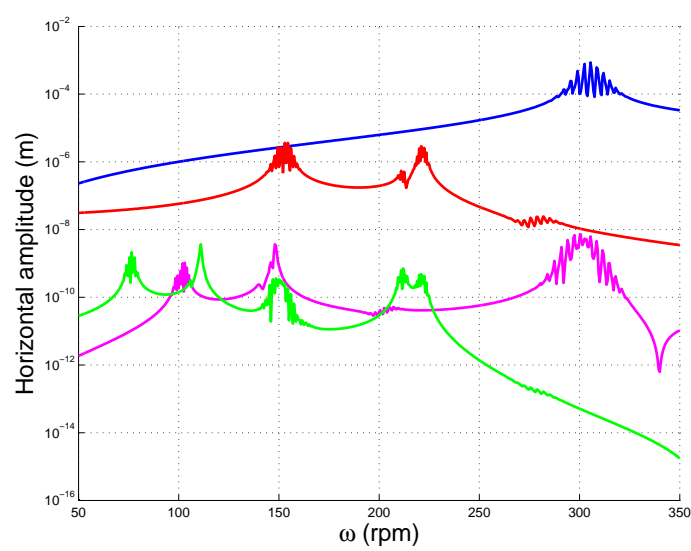

(c)

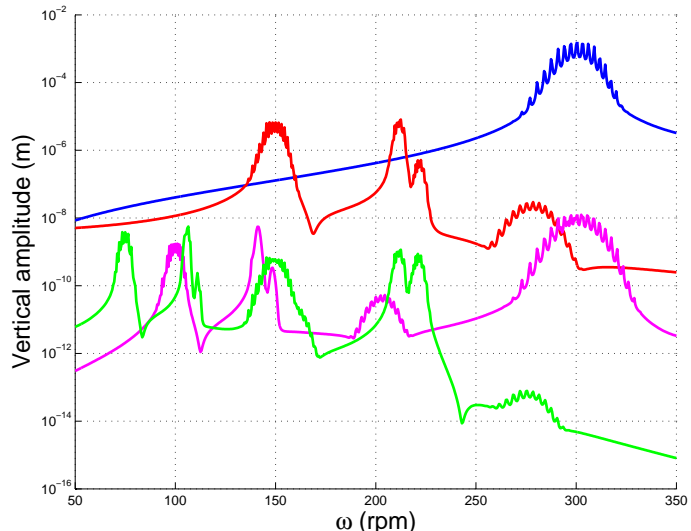

(b)

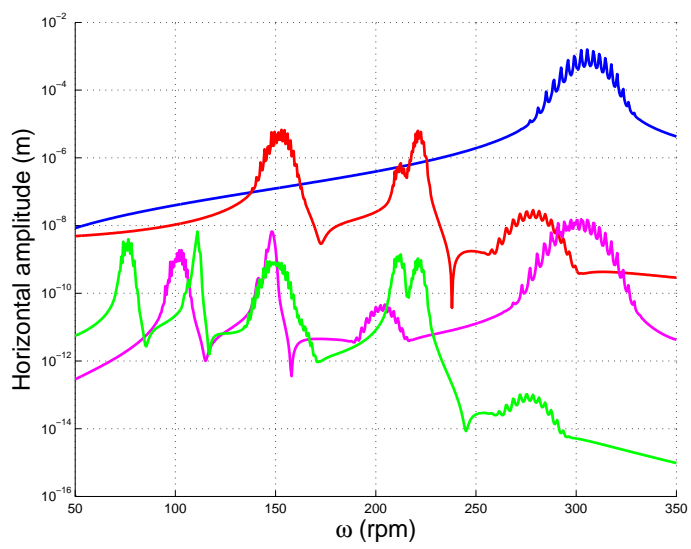

(d)

Figure 6: PC order=30; vertical response of order 1 to 4 at the mid-span : mean (a), standard deviation (b); horizontal response of order 1 to 4 at the mid-span : mean (c), standard deviation (d), (blue $=1 \times$ response, red $=2 \times$ response, magenta $=3 \times$ response, green $=4 \times$ response) 


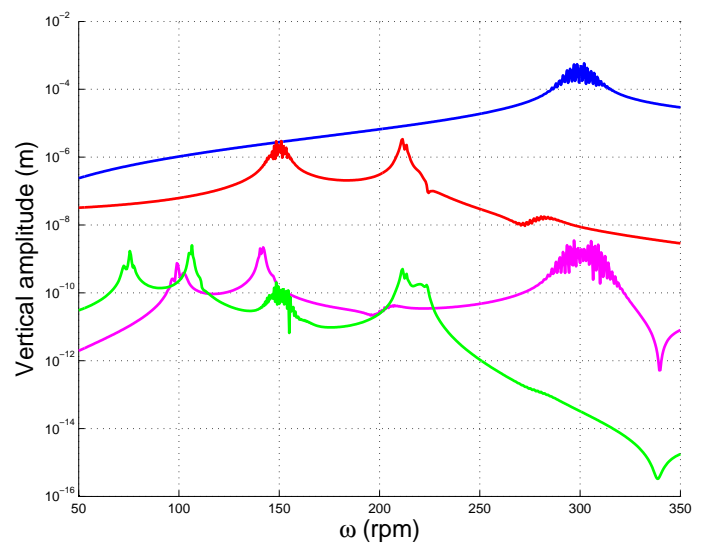

(a)

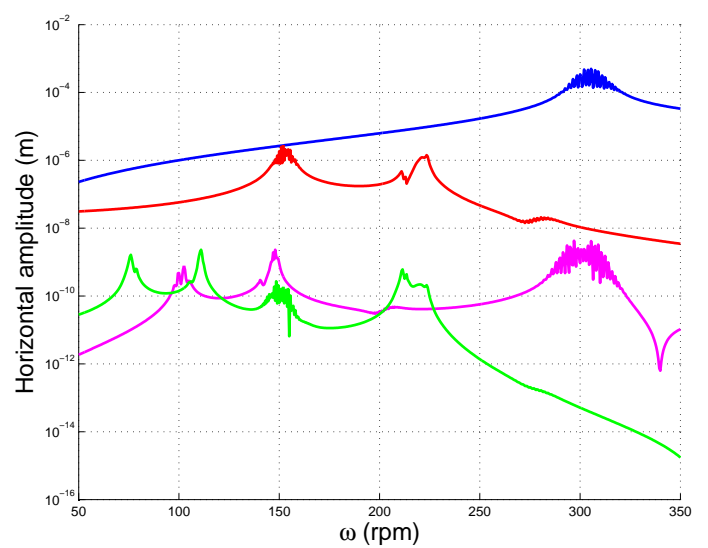

(c)

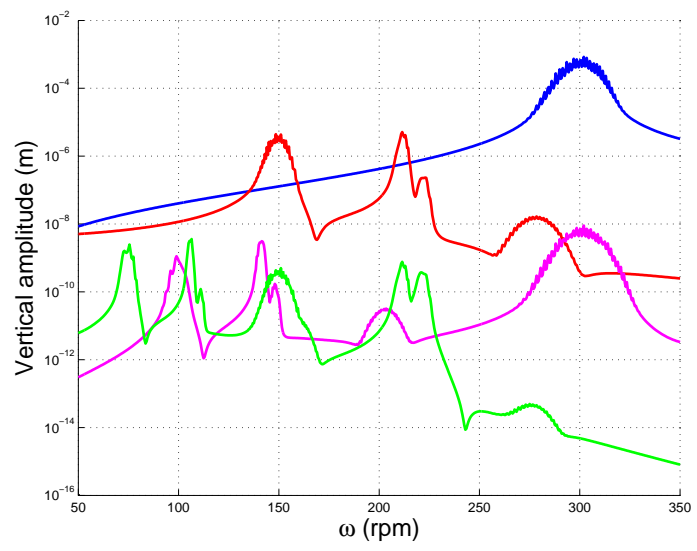

(b)

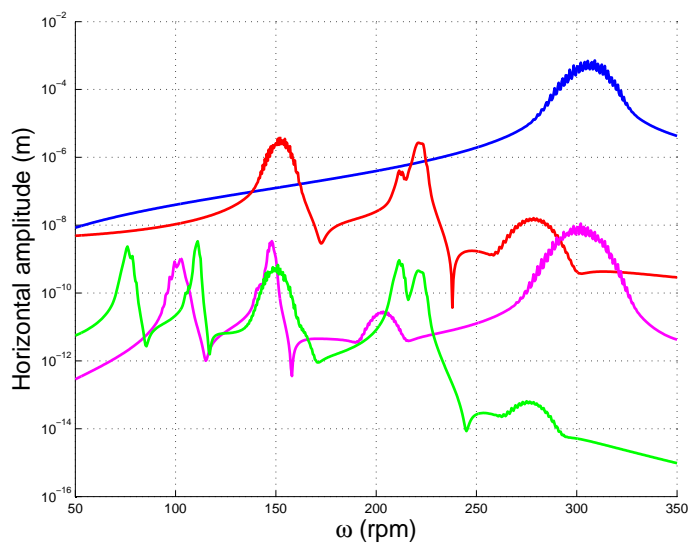

(d)

Figure 7: PC order=200; vertical response of order 1 to 4 at the mid-span : mean (a), standard deviation (b); horizontal response of order 1 to 4 at the mid-span : mean (c), standard deviation (d), (blue $=1 \times$ response, red $=2 \times$ response, magenta $=3 \times$ response, green $=4 \times$ response) 


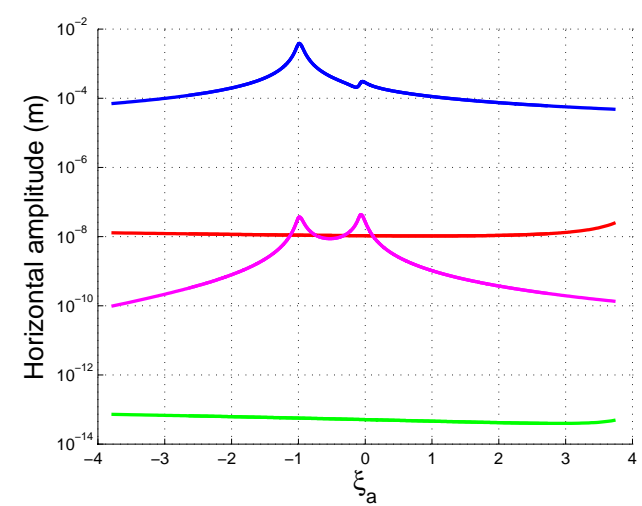

(a)

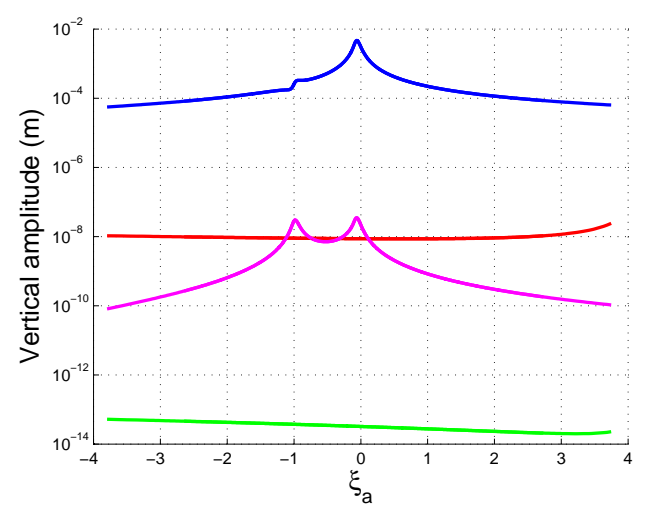

(b)

Figure 8: Distribution of the horizontal (a) and vertical (b) responses of order 1 to 4 at the mid-span at $\omega=300 \mathrm{rpm}$, (blue $=1 \times$ response, red $=2 \times$ response, magenta $=3 \times$ response, green $=4 \times$ response) 


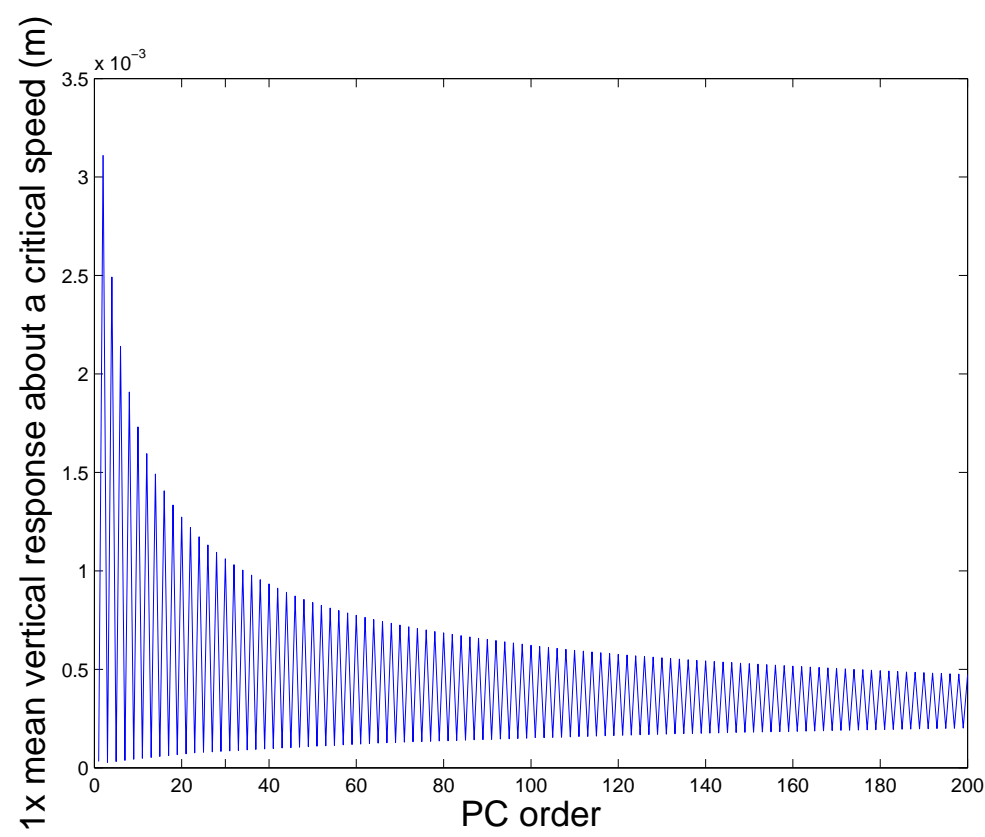

Figure 9: $1 \times$ Mean vertical response vs. PC order at 300.3rpm 


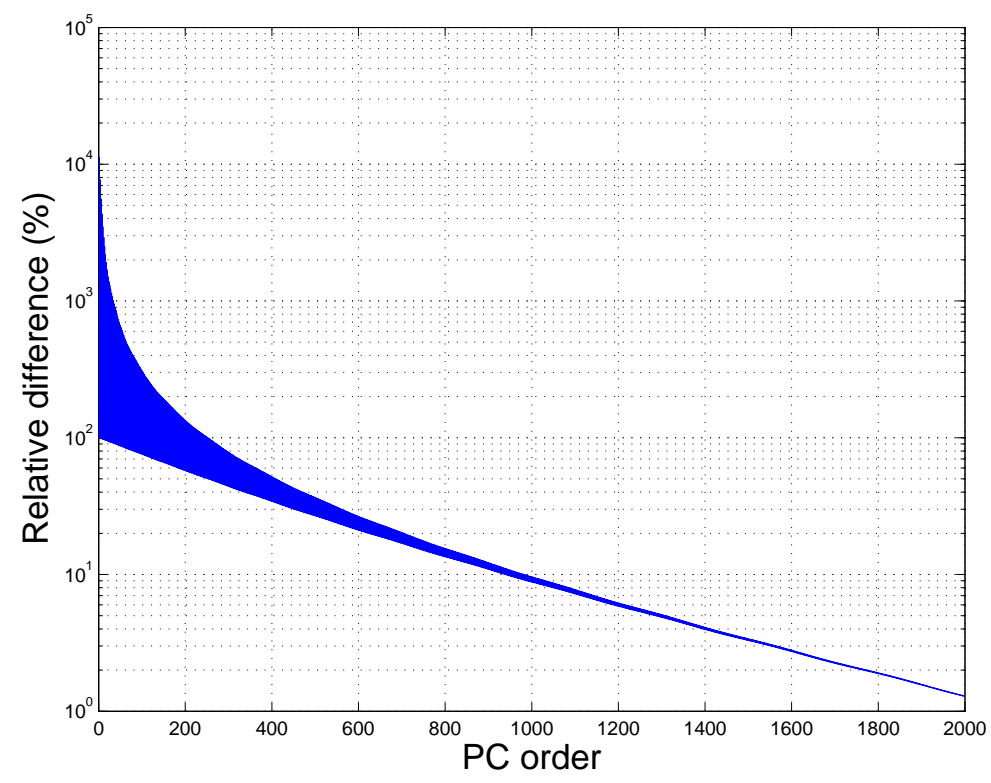

Figure 10: Relative difference $\mathcal{D}(P)$ vs. PC order 


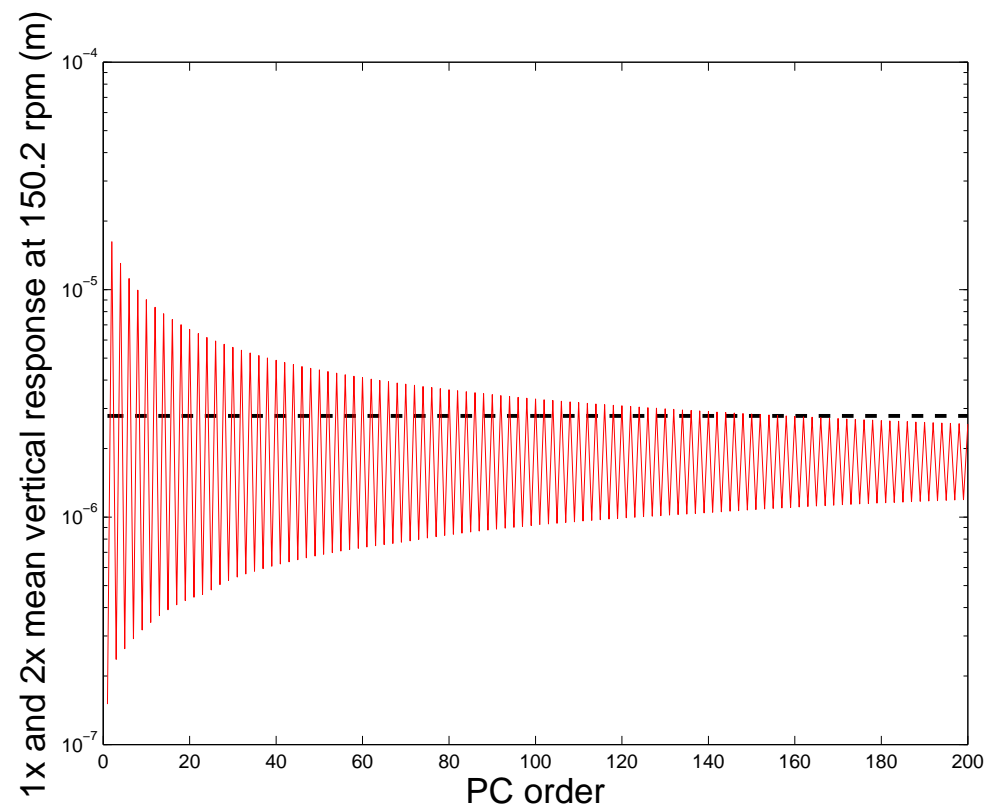

Figure 11: $1 \times$ (dashed line) and $2 \times($ solid line $)$ mean vertical response vs. PC order at $150.2 \mathrm{rpm}$ 


\section{List of Tables}

1 Value of the physical parameters . . . . . . . . . . . . . . . . . 29

2 Value of the physical parameters for the asymmetric coupling . . . . . . . . . . . 30 


\begin{tabular}{ccc}
\hline Notation & Description & Value \\
\hline$R$ & radius of the rotor shaft & $0.02 \mathrm{~m}$ \\
$L$ & length of the rotor shaft & $0.7 \mathrm{~m}$ \\
$R_{D}$ & outer radius of the disk & $0.3 \mathrm{~m}$ \\
$h_{D}$ & thickness of the disk & $0.02 \mathrm{~m}$ \\
$E$ & Young's modulus of elasticity & $2.110^{11} \mathrm{~N} \cdot \mathrm{m}^{-2}$ \\
$G$ & shear modulus & $7.710^{10} \mathrm{~N} \cdot \mathrm{m}^{-2}$ \\
$\rho$ & density & $7800 \mathrm{~kg} \cdot \mathrm{m}^{-3}$ \\
$\nu$ & Poisson ratio & 0.3 \\
$K_{x}$ & stiffness of supports (vertical direction) & $210^{6} \mathrm{~N} \cdot \mathrm{m}^{-1}$ \\
$K_{y}$ & stiffness of supports (horizontal direction) & $2.210^{6} \mathrm{~N} \cdot \mathrm{m}^{-1}$ \\
\hline
\end{tabular}

Table 1: Value of the physical parameters 


\begin{tabular}{cc}
\hline Notation & Value \\
\hline$k_{x x}$ & $4.410^{4} \mathrm{~N} . \mathrm{m}^{-1}$ \\
$k_{y y}$ & $3.5210^{4} \mathrm{~N} . \mathrm{m}^{-1}$ \\
$k_{\phi \phi}$ & $1.4110^{4} \mathrm{~N} . \mathrm{m}^{-1}$ \\
$k_{\theta \theta}$ & $1.7610^{4} \mathrm{~N} . \mathrm{m}^{-1}$ \\
\hline
\end{tabular}

Table 2: Value of the physical parameters for the asymmetric coupling 